Int. J. Dev. Biol. 57: 837-844 (2013)

doi: $10.1387 / \mathrm{ijdb} .130044 \mathrm{md}$

\title{
Insulin-like growth factor 1 acts as an autocrine factor to improve early embryogenesis in vitro
}

\author{
CHARMAINE J. GREEN and MARGOT L. DAY* \\ Department of Physiology, Bosch Institute, University of Sydney, Sydney, Australia
}

\begin{abstract}
Consideration of embryo-derived growth factors, such as IGF1, is important when culturing an embryo in an in vitro fertilization (IVF) setting, or when studying the effect of growth factors on embryo development in vitro. Addition of IGF1 to the culture medium has been reported to cause a range of developmental responses in preimplantation mouse embryos. This variability may be due to culture of embryos in suboptimal culture media and at different culture densities/ volumes. This study examined the role of exogenous and autocrine IGF1 on mouse preimplantation development in vitro, by treatment of embryos with an IGF1R neutralising antibody (IGF1R nAb) under low density $(1$ embryo/100 $\mu \mathrm{l})$ or high density $(1$ embryo/1 $\mu \mathrm{l})$ culture conditions. At low density, IGF1R $\mathrm{nAb}$ reduced development to the blastocyst stage, hatching, and total cell numbers in blastocysts and increased the number of apoptotic cells in blastocysts, suggesting that autocrine IGF1 signalling is occurring, even at low density. This signalling is independent of IGF1 present in the zona pellucida, since culturing embryos in the absence of their zona pellucida had no effect on blastocyst development. Addition of $10 \mathrm{ng} / \mathrm{ml}$ IGF1 increased blastocyst development at low density, but decreased hatching at high density. Similarly, high levels of exogenous IGF1 at low density decreased hatching. IGF1 appears to play a role in cell survival and treatment of blastocysts with IGF1 increased Akt phosphorylation. The IGF1R antagonist picropodophyllin was also used in this study, but was found to have non-specific effects on the mitotic spindle. In conclusion, IGF1 is an important growth factor for the improvement of preimplantation development; however, for optimal development the total amount of IGF1 present must be tightly controlled.
\end{abstract}

KEY WORDS: IGF1, embryogenesis, autocrine growth factor

\section{Introduction}

The preimplantation embryo develops in luminal fluid secreted by the maternal reproductive tract. This fluid contains a large number of maternal and embryo-derived growth factors (reviewed in (Hardy and Spanos, 2002)). Interestingly, preimplantation embryo development can occur in the absence of such factors, in a simple chemically defined medium (Whitten, 1956). However, embryos that develop in this way exhibit a slower rate of cleavage and a higher amount of developmental arrest compared to embryos that develop in vivo (reviewed in Summers and Biggers, 2003). Although there has been substantial improvement in media for mammalian preimplantation embryo culture, even the best media currently used may not be optimal and inevitably cause imbalances and stress to the embryos (reviewed in Summers and Biggers, 2003). Growth factors present in vivo may enable embryos to attain optimum development not possible in simple, chemically defined medium. Thus the inclusion of one or more growth factors in culture media and their concentration must be carefully considered. The benefit of the presence of growth factors in culture media is highlighted by the culture of embryos in high density compared to low density. Under high density embryo culture conditions, where the embryo is cultured in a small volume of medium or at high embryo concentrations, embryo-derived

Abbreviations used in this paper: BSA, Bovine serum albumin; Hepes mod-HTF, Hepes buffered modified synthetic human tubal fluid; hCG, Human chorionic gonadotropin; IGFBP, IGF binding protein; IGF1, insulin-like growth factor 1; IGF1R, Insulin-like growth factor 1 receptor; IGF1R nAb, Insulin-like growth factor 1 receptor neutralising antibody; IVF, in vitro fertilisation; KSOM, Potassium simplex optimized medium; KSOM/AA, Potassium simplex optimized medium/ amino acids; MAPK, Mitogen activated protein kinase; M II, Mitosis phase 2; NS, No significant difference; PAF, Platelet activating factor; PBS, Phosphate buffered saline; PDGF $\alpha$, Platelet-derived growth factor- $\alpha$; PI3K, Phosphoinositide 3-kinase; PMS, Pregnant mares' serum gonadotropin; PPP, Picropodophyllin; TGF- $\alpha$, Transforming growth factor- $\alpha$.

\footnotetext{
*Address correspondence to: Margot Day. Department of Physiology, K25 - Medical Foundation Building, University of Sydney, NSW, 2006, Australia. Tel: +6-12-9036-3312 Fax: +6-12-9036-3316. e-mail: margotd@ physiol.usyd.edu.au
}

Supplementary Material (two figures and one video) for this paper is available at: http://dx.doi.org/10.1387/ijdb.130044md

Accepted: 26 September 2013. Final, author-corrected PDF published online: 22 January 2014. Edited by: Sally Dunwoodie.

ISSN: Online 1696-3547, Print 0214-6282

(C) 2014 UBC Press

Printed in Spain 
growth factors become concentrated in the medium and then act on the preimplantation embryo to improve development (Lane and Gardner, 1992, Melin et al., 2009, Paria and Dey, 1990). While under low density culture conditions, where the embryo is cultured in a large volume of medium, embryo-derived growth factors are diluted resulting in poor development (O'Neill, 1997, O'Neill, 1998).

IGF1 is one such growth factor which is secreted by the preimplantation embryo and IGF1 and its receptor are present from the oocyte stage and throughout the preimplantation period (Inzunza et al., 2010). In the mouse and rabbit, IGF1 binds to the zona pellucida, the extracellular matrix which surrounds the preimplantation embryo (Herrler et al., 1997, Smith et al., 1993). Removal of the zona pellucida decreases blastocyst development (Suzuki et al., 1995), suggesting that IGF1 found in the zona pellucida may contribute to the available autocrine IGF1.

Although there was a great deal of work on the action of IGF1 in the early embryo in the late 80 s and early 90 s, this work occurred before the culture density dependence of embryos was realised and when only suboptimal culture media was available. As a result, a range of developmental responses by preimplantation mouse embryos to the addition of IGF1 to the culture medium has been reported. These include increased blastocyst formation, protein synthesis and the number of cells in the inner cell mass or a decrease in apoptosis (Brison, 2000, Harvey and Kaye, 1992, Markham and Kaye, 2003). While others have reported no accelerated rate of development (Heyner et al., 1993, Paria and Dey, 1990), or only an increased rate of hatching (Heyner et al., 1993). Firstly, the interpretation of a negative result needs to be considered carefully as suboptimal culture media has been directly linked to decreases in Igf1, Igf1r, Igf2 and Igf2r expression (Ho et al., 1995). Secondly, some studies reported that IGF1 improved development at high embryo concentrations (Harvey and Kaye, 1992, Markham and Kaye, 2003), in which activation of autocrine signalling pathways would be expected. Thus the question of whether this positive effect of IGF1 was a result of synergy between growth factors remains. None the less, at least some of the variability reported, in response to IGF1, may be due to the presence of endogenous IGF1 and other embryo-derived growth factors.

This discrepancy in the literature of the effect of exogenous IGF1 warrants new investigation into IGF1 action. In the present study, experiments have been carefully conducted in order to elucidate the role of IGF1 in mouse preimplantation development. The current study utilised potassium simplex

Fig. 1. Exogenous IGF1 improves development to the blastocyst stage at low density, but not at high density. (A) Development of zygotes to the blastocyst stage and proportion of blastocysts hatching after culture at low density $(1$ embryo/100 $\mu$ l) or in groups at high density (1 embryo/1 $\mu$ l). (B) Schematic showing the two culture densities: low density (1 embryo/100 $\mu$ l), individual culture and high density (1 embryo/1 $\mu$ l), group culture. Development of zygotes to the 2, 4-8, morula and blastocyst stage after culture at low density at (C) 1 embryo/100 $\mu$ lor (D) 1 embryo/300 $\mu$ l, in the absence or presence of $10 \mathrm{ng} / \mathrm{m} / \mathrm{IGF}$. The results are displayed as the percentage of embryos developed to each stage or hatched from the zona pellucida, pooled from at least three experiments (n values in parentheses). Chi-square analysis was used to compare the control to the treatment groups. ${ }^{*}$ indicates $\mathrm{P}<0.05 * *$ indicates $\mathrm{P}<0.01$.

optimised medium (KSOM), an optimal culture medium that provides excellent support for mouse embryo development (Erbach et al., 1994). KSOM supports normal expression of some genes involved in metabolism, transcription and cell proliferation including Igf1, Igf1r, Igf2 and $\operatorname{lgf} 2 r$ (Ho et al., 1995). In the present study the density of culture was varied to determine the role of a physiological concentration of IGF1 on preimplantation embryo development from the zygote stage. Furthermore, the involvement of autocrine IGF1R signalling in preimplantation development was investigated using inhibitors of the IGF1R, namely picropodophyllin (PPP) and an IGF1R neutralising antibody (IGF1RnAB). Since activation of the IGF1R has been shown to decrease apoptosis in preimplantation embryos (Spanos et al., 2000) the effect of IGF1 on apoptosis and pAkt activation were examined.

\section{Results}

\section{Effect of IGF1 on development of preimplantation embryos cultured at different densities}

Since the discrepancies in previously reported studies of IGF1 on development may be due to the lack of consideration for culture density, the effect of IGF1 on preimplantation embryos was assessed

A

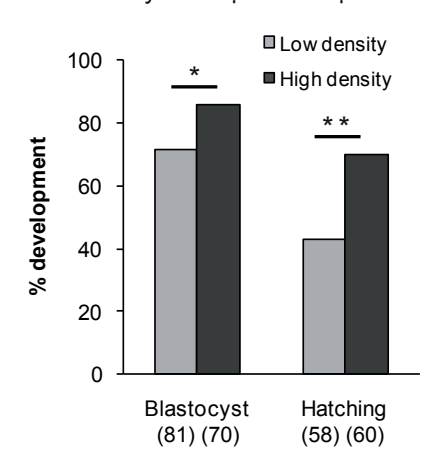

C 1 embryo/ $100 \mu \mathrm{l}$

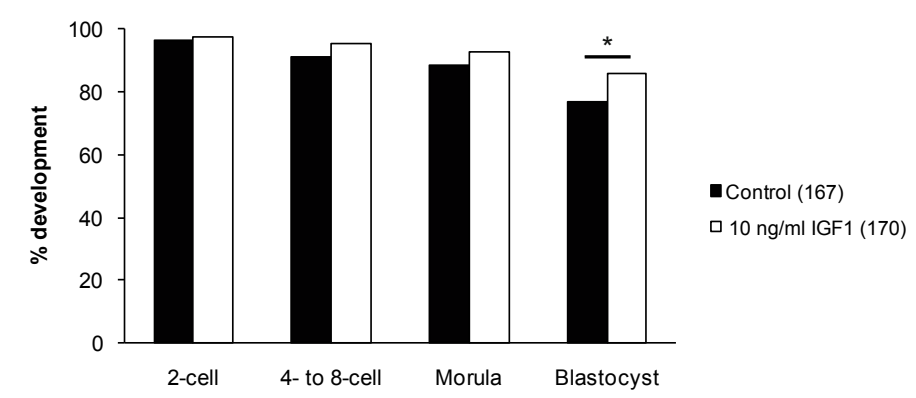

D 1 embryo/300 $\mu \mathrm{l}$

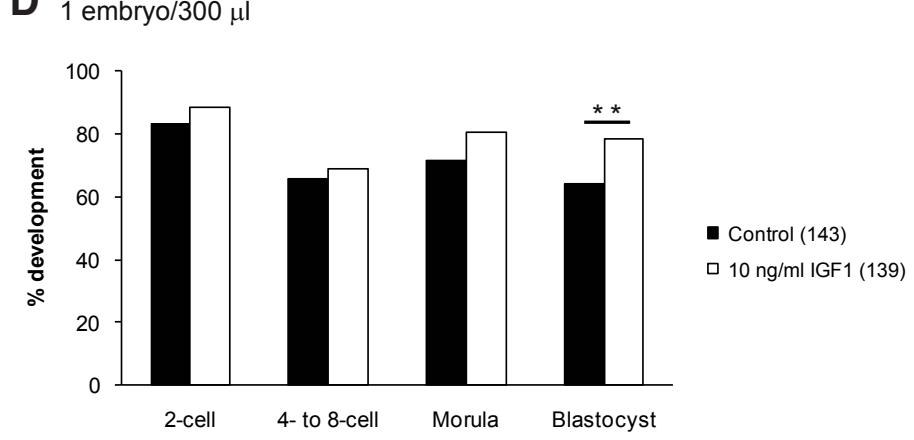

B Low density vs High density

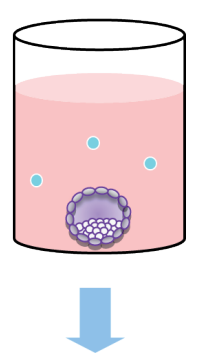

Poor development
- Autocrine growth factor

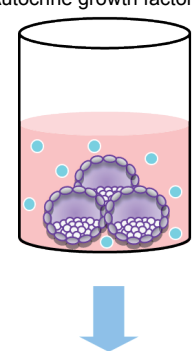

Good development 
at high density, when autocrine growth factors, including IGF1 are concentrated, and at low density, when autocrine growth factors are diluted. In the present study embryo viability was assessed in terms of blastocyst development, hatching from the zona pellucida, cell numbers and apoptosis. Firstly, we confirmed that culture of embryos at high density significantly increased the percentage of embryos that developed to the blastocyst stage $(P<0.05)$ and that hatched from their zonae pellucidae $(P<0.01)$, compared to the paired low density control (Fig. 1A).

Culture of zygotes individually in $100 \mu \mathrm{l}$ or $300 \mu \mathrm{l}$ of medium in the presence of $10 \mathrm{ng} / \mathrm{ml}$ IGF1 caused a significant increase in development to the blastocyst stage (Fig. 1C; $P<0.05$, Fig. 1D; $P<0.01)$. No improvement in development was observed prior to the blastocyst stage. Furthermore no effect on hatching was observed in either $100 \mu \mathrm{l}(P=0.487$, data not shown) or $300 \mu \mathrm{l}(P=0.466$, data not shown). The number of cells in blastocysts cultured in $100 \mu \mathrm{l}$ of medium was also determined. IGF1 had no effect on total cell numbers $(P=0.685 ; n \geq 101$, data not shown) or cell numbers in the ICM ( $P=0.065 ; \mathrm{n} \geq 39$, data not shown). Furthermore, IGF1 had no effect on the number of apoptotic cells in blastocysts cultured at low density ( $P=0.478 ; \mathrm{n} \geq 88$, data not shown).

\section{Effect of high concentrations of IGF1 on blastocyst development}

Treatment of zygotes cultured at high density with $10 \mathrm{ng} / \mathrm{ml} \mathrm{IGF1}$ had no effect on development to the blastocyst stage (Fig. 2B; $P=0.820)$, or total cell numbers in blastocysts $(P=0.127, \mathrm{n} \geq 42$, data not shown). Embryos treated with IGF1 (10 ng/ml) at high density however, had a decreased level of hatching compared to the control (Fig. $2 A ; P<0.05)$ but this was not due to an increased number of apoptotic cells in blastocysts ( $P=0.796 ; n \geq 42$, data not shown).

Previously, treatment of mouse blastocysts with $100 \mathrm{ng} / \mathrm{ml} \mathrm{IGF1}$ was shown to down regulate the IGF1R (Chi et al., 2000, Smith et al., 1993) and induce apoptosis (Chi et al., 2000). The effect of 30 and $100 \mathrm{ng} / \mathrm{ml} \mathrm{IGF1}$ on development to the blastocyst stage, hatching, cell numbers and apoptosis at low density was investigated. Neither 30 nor 100 ng/ml IGF1 improved blastocyst development (Fig. 2B; $P=0.721$; and $P=0.616$ ). Instead, both concentrations decreased hatching compared to the control (Fig. 2B; $P<0.01) .30$ and $100 \mathrm{ng} / \mathrm{ml}$ IGF1 had no effect on total cell numbers $(P=0.804$; $\mathrm{n}=19$, data not shown), cell numbers in the $\operatorname{ICM}(P=0.753 ; n \geq 9$, data not shown), or on the number of apoptotic cells in blastocysts $(P=0.669 ; \mathrm{n}=19$, data not shown).

\section{Role of autocrine IGF on preimplantation embryo development in vitro}

The role of autocrine IGF1 signalling during early embryo development in vitro was examined using an IGF1R $n A b$ to specifically inhibit IGF1 binding to its receptor. Embryos were cultured in the presence or absence of IGF1R nAb (0.1-2 ng/ml IGF1R nAb) at low and high density to specifically inhibit IGF1R signalling. At low density $(1$ embryo/100 $\mu \mathrm{l}) 2 \mathrm{ng} / \mathrm{ml}$ IGF1 $\mathrm{R}$ nAb reduced development to the blastocyst stage (Fig. 3A) and hatching (Fig. 3B) compared to the control $(P<0.05)$. This loss of viability occurred from compaction onwards (data not shown). 0.1-1 ng/ml IGF1R nAb had no effect on blastocyst development (Fig 3A) or hatching (Fig. 3B). Total cell numbers in blastocysts cultured at low density were decreased by treatment with 1 and 2 ng/ml IGF1R nAb (Fig. 3C; $P<0.05) .0 .5$ ng/ $\mathrm{ml}$ and $0.1 \mathrm{ng} / \mathrm{ml}$ IGF1R $\mathrm{nAb}$ had no effect on cell numbers (Fig. 3C; $P>0.05) .2 \mathrm{ng} / \mathrm{ml}$ IGFR nAb increased the number of apoptotic cells in blastocysts cultured at low density (Fig. 4B; $P<0.001$ ).

Culture of embryos at low density ( 1 embryo/100 $\mu$ l) with $2 \mathrm{ng} /$ $\mathrm{ml}$ goat IgG had no effect on development to the blastocyst stage $(P=0.369 ; \mathrm{n} \geq 51$, data not shown), hatching $(P=0.175 ; \mathrm{n} \geq 33$, data not shown) or cell numbers ( $P=0.101 ; n \geq 31$, data not shown). Treatment of embryos cultured at high density with $2 \mathrm{ng} / \mathrm{ml}$ IGF1R nAb had no effect on blastocyst development, hatching, cell numbers, or apoptosis (data not shown).

\section{Effect of IGF1 on Akt phosphorylation in blastocysts}

The best defined pathways by which IGF1R signalling can prevent apoptosis are mediated by phosphoinositide 3-kinase (PI3K) and Akt. Since treatment of embryos cultured at low density with $2 \mathrm{ng} / \mathrm{ml} \mathrm{IGF1R} \mathrm{nAb} \mathrm{increased} \mathrm{apoptosis} \mathrm{in} \mathrm{blastocysts,} \mathrm{the} \mathrm{effect} \mathrm{of}$ IGF1 on Akt phosphorylation was investigated by western blotting. Treatment of mouse blastocysts with $100 \mathrm{ng} / \mathrm{ml} \mathrm{IGF1} \mathrm{increased}$ phosphorylation of Akt after 10 minutes (Fig. 4C).

\section{Effect of IGF1R neutralising antibody on blastocyst develop- ment in the absence of the zona pellucida}

The role of the zona pellucida in the autocrine action of IGF1 on development was investigated as IGF1 has been shown to bind to the zona pellucida of mouse embryos (Smith et al., 1993). Culturing

A 1 embryo/1 $\mu \mathrm{l}$

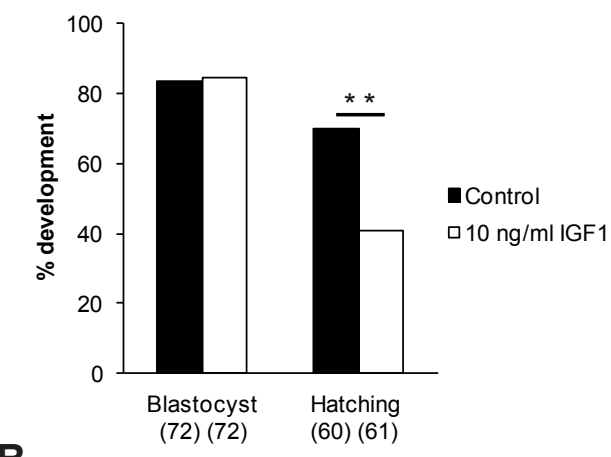

1 embryo/100 $\mu \mathrm{l}$

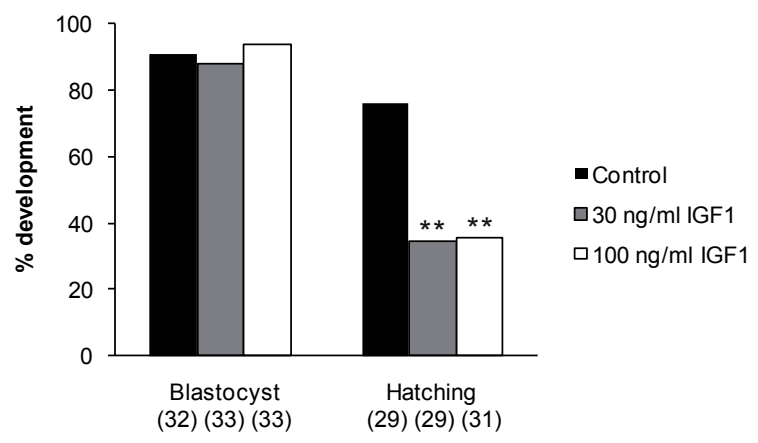

Fig. 2. High concentrations of IGF1 decrease blastocyst hatching Development of zygotes to the blastocyst stage and proportion of blastocysts hatching after culture (A) in the absence or presence of $10 \mathrm{ng} / \mathrm{ml}$ IGF1 in groups at high density (1 embryo/1 $\mu$ l) and (B) individually at low density (1 embryo/100 $\mu$ l) in the absence or presence of 30 or $100 \mathrm{ng} / \mathrm{ml}$ IGF1. The results are displayed as the percentage of embryos developed to blastocyst stage or hatching, pooled from at least three experiments (n values in parentheses). Chi-square analysis was used to compare the control to the treatment groups. ${ }^{* *}$ indicates $\mathrm{P}<0.01$. 
embryos at low density ( 1 embryo/100 $\mu \mathrm{l}$ ) in the absence of their zonae pellucidae had no effect on development to the blastocyst stage (Fig. 5; $P=0.456$ ). Furthermore, the presence of $2 \mathrm{ng} / \mathrm{ml}$ IGF1R $\mathrm{nAb}$ still decreased blastocyst development in zona free embryos cultured at low density (Fig. 5; $P<0.05$ ).

\section{Effect of picropodophyllin on blastocyst development in vitro}

The effect of the IGF1R antagonist picropodophyllin (PPP) was investigated as it has been shown to completely inhibit mouse em- bryo development (Inzunza et al., 2010). The culture of embryos at both low and high density in the presence of varying concentrations of PPP inhibited development and reduced cell numbers (Fig. S1). Since blocking the IGF1R with the neutralising antibody and PPP produced differing results, we investigated whether the greater potency of PPP was due to its non-specific action on the mitotic spindle as reported in other cell types (Kelleher, 1977, Loike et al., 1978). 26 out of 28 zygotes arrested during mitosis when cultured for 24 hours in the presence of $0.25 \mu \mathrm{M}$ PPP (Fig. 6A).

A 1 embryo/100 $\mu \mathrm{l}$

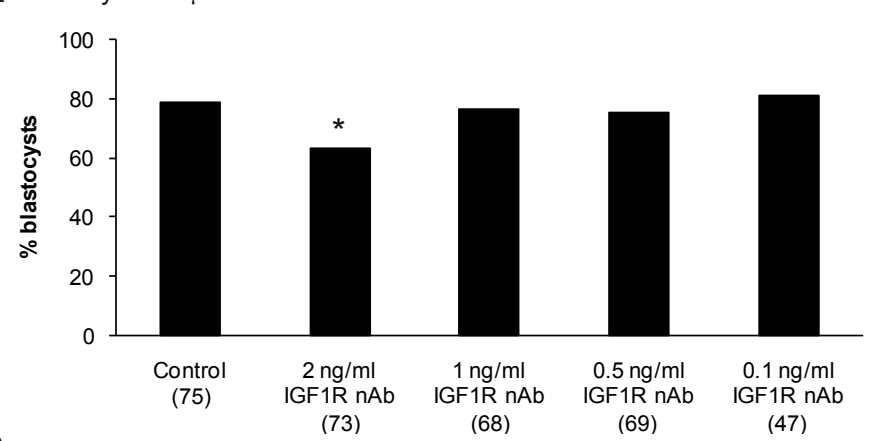

A

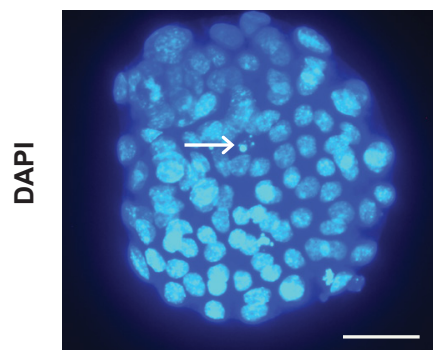

B

1 embryo/100 $\mu \mathrm{l}$

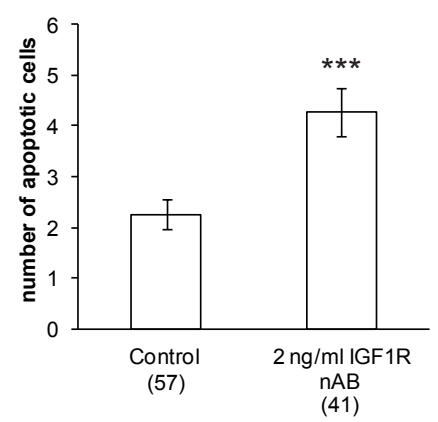

C
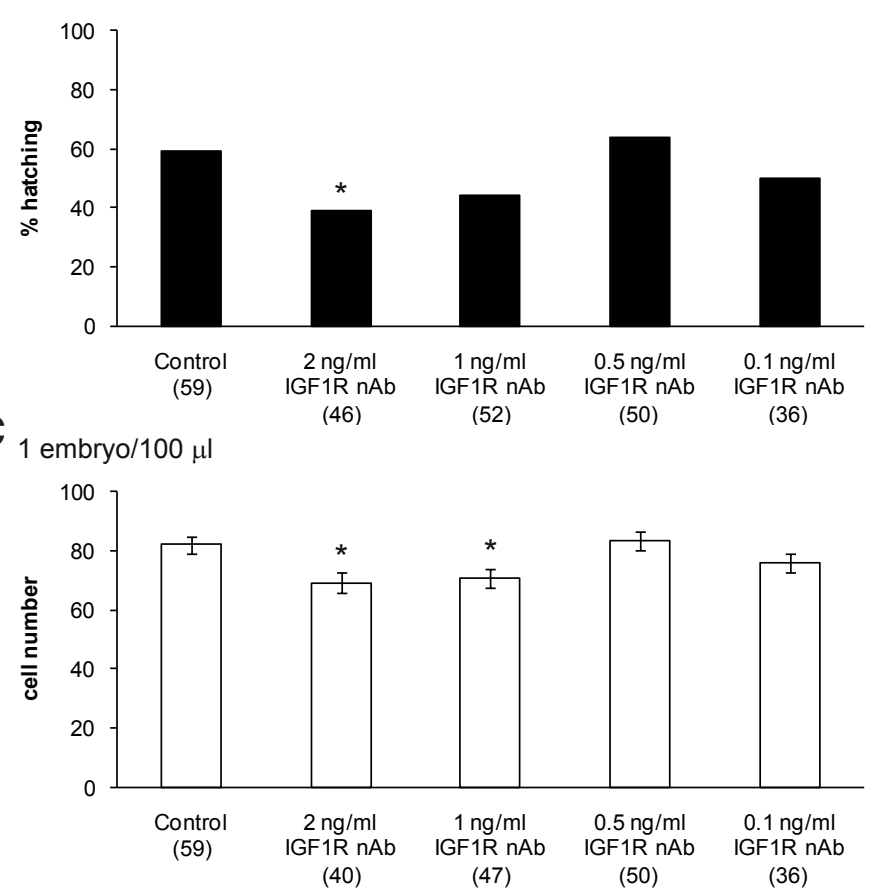

c

(40)

(47)

$(50)$

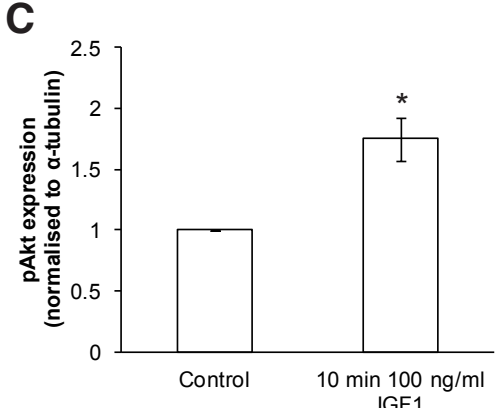

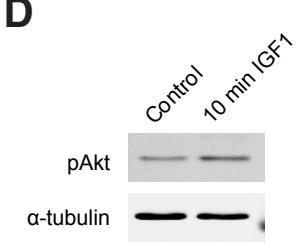

Fig. 3 (left). IGF1R neutralising antibody decreases development to the blastocyst stage and blastocyst cell numbers. Zygotes were cultured at low density $(1 \mathrm{embryo} / 100 \mu \mathrm{l})$ in the presence or absence of $0.1-2 \mathrm{ng} / \mathrm{ml} / \mathrm{GF} 1 \mathrm{R} \mathrm{nAb}$. The results are displayed as (A) the percentage of embryos developed to blastocyst stage, (B) the percentage of blastocysts hatching and (C) the average total number of cells per blastocyst \pm SEM, pooled from at least three experiments (n values in parentheses). Chi-square analysis was used to compare the development and hatching rate of the control to the treatment groups. One-way ANOVA followed by Dunnett's multiple comparisons test was used to compare cell numbers of the control to the treatment groups. ${ }^{*}$ indicates $\mathrm{P} \leq 0.05$.

Fig. 4 (right). IGF1 decreases apoptosis by activation of Akt. (A) Representative images of blastocysts cultured from the zygote stage in the presence or absence of $2 \mathrm{ng} / \mathrm{ml}$ IGF1R nAb. Arrows show examples of apoptotic cells. Scale bars represent 100 um. (B) The average number of apoptotic cells per blastocyst \pm SEM, pooled from at least three experiments (n values in parentheses). (C) Effect of $100 \mathrm{ng} / \mathrm{ml} / \mathrm{GF} 1 \mathrm{on}$ phospho-Akt levels in blastocysts, detected by western blotting. Data are average $p$-Akt band intensities $( \pm S E M, n=3)$ normalised to $\alpha$-tubulin and are expressed relative to the control. (D) Representative western blot of blastocysts exposed to $100 \mathrm{ng} / \mathrm{ml}$ IGF1 for 10 minutes. Western blots were probed for anti-p-Akt (upper panel) and anti- $\alpha$-tubulin (lower panel). T-test analysis was used to compare the control to the treatment groups. ${ }^{*}$ indicates $\mathrm{P}<0.05 * * *$ indicates $\mathrm{P}<0.001$. 
1 embryo/100 $\mu \mathrm{l}$

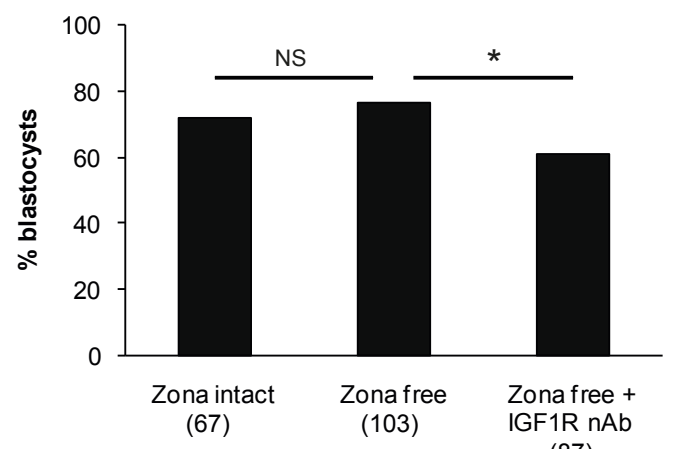

(87)

Fig. 5. Culturing embryos without their zona pellucida has no effect on development to the blastocyst stage. Zygotes were treated with pronase to remove their zonae pellucidae and then cultured at low density (1 embryo/100 ml) with or without the addition of $2 \mathrm{ng} / \mathrm{ml} / \mathrm{GF} 1 \mathrm{R} \mathrm{nAb}$. The results are displayed as the percentage of embryos developed to blastocyst stage, pooled from at least three experiments ( $n$ values in parentheses). The development rates between zona free and zona intact or zona free and zona free with $2 \mathrm{ng} / \mathrm{ml}$ IGF1R nAb were compared by chi-square analysis. NS indicates not significant, ${ }^{*}$ indicates $\mathrm{P}<0.05$.

This compares to 29 out of 30 control embryos that had reached the 2-cell stage. Incubation of metaphase II oocytes in medium containing $0.5 \mu \mathrm{M}$ PPP over a period of 6 hours caused disruption of spindles and dispersal of the chromosomes throughout $100 \%$ of oocytes ( $n=28$, Fig. 6B, 6C and Supplementary video S1). All of the control oocytes $(n=27)$, not exposed to PPP, maintained normal spindle structure and had chromosomes that were aligned along the metaphase plate (Fig. 6B).

\section{Discussion}

In the present study the culture of embryos at high density significantly improved the proportion of blastocysts developing and hatching from their zonae pellucidae, compared to those cultured at low density. It is well established that increasing embryo culture density improves embryo viability due to the presence of embryo-derived growth factors that are beneficial to development (reviewed in (O'Neill, 2008)). Under low density culture conditions, embryo-derived growth factors are diluted resulting in poor embryo development (O’Neill, 1997, O’Neill, 1998). Increasing embryo culture density, by culturing embryos in groups (Lane and Gardner, 1992, O'Neill, 1997, Paria and Dey, 1990), or by reducing culture volume (Melin et al., 2009) results in improved embryo development due to the concentration of embryo-derived growth factors. IGF1 is one growth factor that may contribute to the improved development seen at high density, as IGF1 and its receptor are present from the oocyte stage and throughout the preimplantation period (Inzunza et al., 2010). However, various effects of IGF1 on the preimplantation embryo have been reported, possibly due to the use of suboptimal culture media which is known to impact on expression of mRNA encoding the IGFs and IGFRs as well as other trophic factors and their receptors, such as the platelet activating factor receptor (Ho et al., 1995, Stojanov et al., 1999, Stojanov and O'Neill, 2001).

In the present study embryos were cultured in potassium simplex optimized medium (KSOM), a culture medium optimised

A
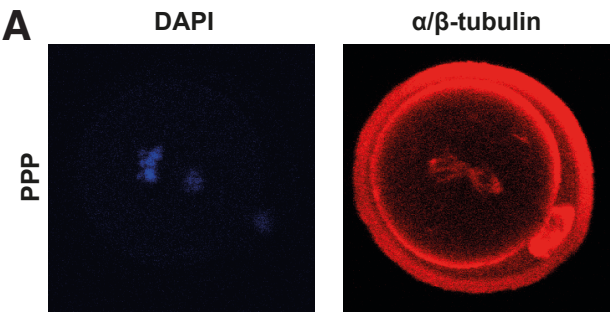

B
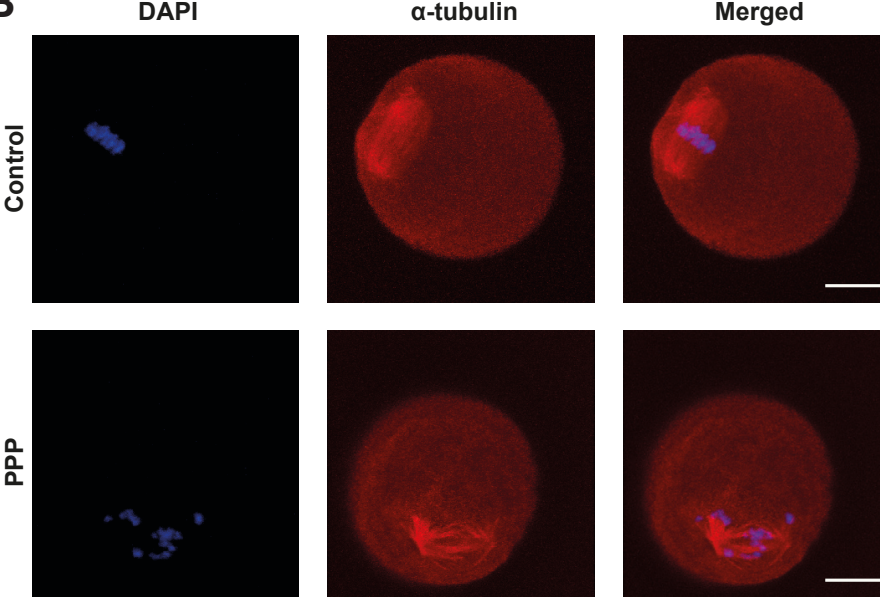

C
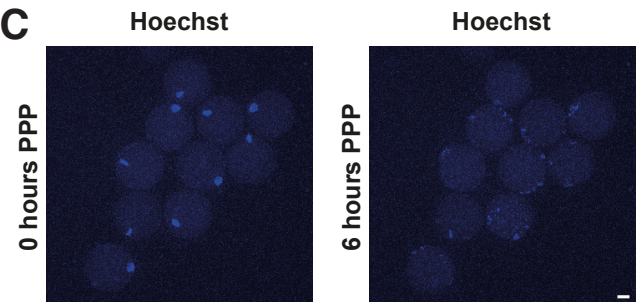

Fig. 6. Picropodophyllin disrupts the spindle in zygotes and oocytes. (A) Representative confocal image of a zygote arrested at the 1-cell stage after treatment with $0.25 \mu \mathrm{M}$ PPP for 24 hours. Anti- $\alpha / \beta$-tubulin staining in red and DAPI staining in blue. (B) Representative 3-D projected confocal image of oocytes cultured in either the absence or presence of $0.5 \mu \mathrm{M}$ PPP for six hours. Anti- $\beta$-tubulin staining in red and DAPI staining in blue. (C) Oocytes cultured in the presence of $0.5 \mu \mathrm{M}$ PPP at zero and six hours. Hoechst staining in blue. Scale bars represent $20 \mu \mathrm{m}$.

for mouse zygote development. At low density (1 embryo/100 or $300 \mu \mathrm{l})$ the presence of IGF1 significantly increased the percentage of embryos that developed to the blastocyst stage, with IGF1 having its affect after the morula stage. This finding correlates with previous studies by Harvey and Kaye (1992) that show increased blastocyst development from compaction onwards. In low density culture $(1$ embryo/ $100 \mu \mathrm{l}$ or $300 \mu \mathrm{l})$, it was assumed that the level of autocrine growth factors was low. However, our results suggest that there was still sufficient embryo-derived IGF1 to impact on development, since the IGF1R nAb, decreased development to the blastocyst stage, hatching and total cell numbers in blastocysts.

High concentrations of IGF1 are known to cause down-regulation of the IGF1R in mouse blastocysts and can be detrimental to development (Chi et al., 2000). This was confirmed by our study, since high concentrations of IGF1 (30 or $100 \mathrm{ng} / \mathrm{ml}$ ) in low density culture and addition of exogenous IGF1 to high density culture reduced blastocyst hatching. The concentrations used in this study are 
similar to the concentration of embryo-derived IGF1 that has been measured in medium after culture of mouse embryos in groups of 30 for 4.5 days (Inzunza et al., 2010). These results suggest that low concentrations of IGF1 improve embryo development in vitro, while high concentrations are detrimental.

A change in blastocyst hatching was one of the predominant responses to IGF1 observed in this study. Inhibition of IGF1R signalling, with the neutralising antibody and down-regulation of the IGF1R by culture in high concentrations of IGF1, both reduced blastocyst hatching. A variety of mechanisms have been proposed to mediate embryo hatching. It is thought that when a crucial cell number is reached thinning of the zona pellucida occurs (Montag et al., 2000). The presence of the IGF1R nAb increased the number of apoptotic cells in blastocysts suggesting that IGF1 stimulates cell survival pathways in the mouse embryo. This was confirmed by our study as treatment of mouse blastocysts with IGF1 increased phosphorylation of Akt. Akt is also implicated in the hatching process since inhibition of Akt in the blastocyst, decreases hatching (Riley et al., 2005). Thus, the decrease in hatching seen when the IGF1R was inhibited may not be due solely to the reduced number of cells in the blastocyst but also due to reduced Akt activity.

Treatment of embryos cultured at high density with IGF1R nAb had no effect on development to the blastocyst stage, hatching, total cell numbers or apoptosis. It is therefore possible that other growth factors present at high density can overcome the loss of IGF1R signalling, possibly by activating the PI3K/Akt pathway. Most of the proposed autocrine factors, such as TGF $\alpha$, PDGF $\alpha$ and PAF (reviewed in (Hardy and Spanos, 2002)) are known to be coupled to PI3K activation and PI3K activation, is promoted by high-density culture, compared to low density (Halet et al., 2008).

IGF1 has been found to bind to the zona pellucida in the mouse and rabbit (Herrler et al., 1997, Smith et al., 1993). Throughout development the rabbit zona pellucida undergoes a structural transformation (Herrler et al., 1997). IGFBP-3 is incorporated into rabbit zona pellucida during this transformation and has been suggested to act here by regulating the transport and availability of IGF to its receptor on the embryo's plasma membrane (Herrler et al., 1997, Herrler et al., 2002). Although evidence suggesting morphological transformation of the mouse zona pellucida is lacking, removal of the zona pellucida in the mouse has been shown to decrease blastocyst development (at 1 embryo/20 $\mu$ l; Suzuki et al., 1995), or have no effect (at 1 embryo/1 $\mu$ ) (Ribas et al., 2006), suggesting a possible role of autocrine signalling by the zona pellucida. In the present study no adverse effect on blastocyst development was seen by zona pellucida removal followed by culture at low density ( 1 embryo/100 $\mu \mathrm{l})$. The IGF1R nAb decreased development to a similar level in both zona free and zona intact embryos, suggesting that IGF1 found in the zona pellucida of the mouse does not have a significant role in autocrine growth factor signalling in vitro.

In addition to the IGF1R nAb the cyclolignan PPP was also used in this study to inhibit IGF1 signalling. PPP inhibits IGF1R autophosphorylation (Girnita et al., 2004) and has been shown to inhibit mouse embryo development at 0.5 and $0.1 \mu \mathrm{M}$ (Inzunza et al., 2010). In the present study PPP caused embryo arrest in a dose dependent manner at both low and high density. These results differ from our IGF1R nAb studies which show that a loss of IGF1R signalling could be overcome by culture at high density. Furthermore, mice carrying null mutations of the IGF1R gene complete gestation (Liu et al., 1993), suggesting that although
IGF1R activation improves development, it is not essential. These discrepancies led us to investigate potential non-specific actions of PPP. Podophyllotoxin (PPT), a cyclolignan related to PPP, displays cytotoxicity due to its interaction with $\beta$-tubulin which prevents microtubule assembly $\left(\mathrm{ID}_{50}=0.6 \mu \mathrm{M}\right)$, causing mitotic arrest in metaphase (Kelleher, 1977, Loike et al., 1978). PPP has a 50 -fold lower inhibitory effect on microtubule assembly $\left(\mathrm{ID}_{50}=\right.$ $30 \mu \mathrm{M})$ (Kelleher, 1977; Loike et al., 1978). Addition of PPP to MII oocytes over a period of 6 hours disrupted the spindle causing the chromosomes to disperse throughout the oocytes. Additionally, zygotes treated with $0.25 \mu \mathrm{M}$ PPP arrested in metaphase and displayed an abnormal spindle. Therefore, we suggest that the detrimental effect of PPP on embryo development is due to non-specific actions, on the mitotic spindle, rather than its ability to prevent IGF1R signalling.

Although it is known that IGF1 improves embryo survival, the signalling pathways activated in the mouse embryo by IGF1 have not been directly determined. In the bovine pre-implantation embryo IGF1 is reported to have a dual role, as a mitogen, promoting cell proliferation through mitogen-activated protein kinase (MAPK) and as a survival factor, inhibiting apoptosis through activation of the PI3K/Akt pathway (reviewed in (Velazquez et al., 2009)). In the mouse embryo, recent evidence suggests that full activation of the IGF1R requires interaction with E-cadherin (Bedzhov et al., 2012). The present study demonstrates for the first time that IGF1 activates the PI3K/Akt pathway in the preimplantation mouse embryo and thereby improves development by inhibiting apoptosis.

In conclusion, by carefully controlling for the effect of embryo culture density and by using a specific IGF1R blocker (IGF1R nAb) this study explains the discrepancies in embryonic responses to IGF1 that have previously been reported and demonstrates an important role for IGF1 in the preimplantation mouse embryo. More importantly, to ensure optimal development, the total amount of IGF1 in the culture media is critical and the presence of other growth factors also needs to be considered.

\section{Materials and Methods}

\section{Media and chemical preparation for embryo collection and culture}

The medium used for embryo collection was Hepes ( $\mathrm{N}-2$ - Hydroxyethylpiperazine-N'-2-ethanesulfonic acid) buffered modified synthetic human tubal fluid medium (Hepes mod-HTF (mM); $\mathrm{NaCl}$ (102), $\mathrm{KCl}(4.6), \mathrm{KH}_{2} \mathrm{PO}_{4}$ (0.4), $\mathrm{MgSO}_{4}(0.2), \mathrm{NaHCO}_{3}$ (4), Na-lactate (21.4), Na pyruvate (0.4), glucose (2.8), $\mathrm{CaCl}_{2}$ (2.04), Hepes (21), $0.06 \mathrm{mg} / \mathrm{ml}$ penicillin, $0.04 \%$ (w/v) phenol red, $\mathrm{pH} 7.4, \sim 300 \mathrm{mosM} / \mathrm{L}$ ) containing $3 \mathrm{mg} / \mathrm{ml}$ bovine serum albumin (BSA; Sigma-Aldrich; St Louis, MO, USA). The medium used for embryo culture was potassium simplex optimized medium (KSOM (mM); $\mathrm{NaCl}$ (95), $\mathrm{KCl}$ (2.5), $\mathrm{KH}_{2} \mathrm{PO}_{4}(0.35), \mathrm{MgSO}_{4}(0.2), \mathrm{NaHCO}_{3}(25), \mathrm{Na}_{2} \operatorname{EDTA}(0.01)$, Na-lactate (10), Na-pyruvate (0.2), L-glutamine (1), glucose (0.2), $\mathrm{CaCl}_{2}$ (2.04), $0.06 \mathrm{mg} / \mathrm{ml}$ penicillin, $0.04 \%$ (w/v) phenol red, pH 7.4; 260 mosM/L) containing $1 \mathrm{mg} /$ $\mathrm{ml}$ BSA. Media was made up from stock solutions prepared from reagents of tissue culture grade (Sigma-Aldrich).

Recombinant mouse IGF1 (R\&D Systems; Minneapolis, MN USA) was reconstituted at $100 \mu \mathrm{g} / \mathrm{ml}$ in sterile phosphate buffered saline (PBS; AMRESCO; Solon, OH, USA). $2 \mu$ l aliquots were prepared and stored at $-80^{\circ} \mathrm{C}$. Prior to use, aliquots were diluted to $10 \mu \mathrm{g} / \mathrm{ml}$ in sterile PBS and then diluted in KSOM to give the desired final concentration.

Human/mouse IGF1R neutralising antibody (IGF1R nAb; AF-305-NA; R\&D Systems) was reconstituted at $0.2 \mathrm{mg} / \mathrm{ml}$ in PBS. $10 \mu \mathrm{l}$ aliquots were prepared and stored at $-80^{\circ} \mathrm{C}$. IGF1R nAb was diluted in KSOM to a final concentration of 0.1 to $2 \mu \mathrm{g} / \mathrm{ml}$. 
Picropodophyllin (PPP; Sigma-Aldrich) was dissolved in dimethyl sulfoxide (DMSO; Sigma-Aldrich) to a concentration of $5 \mathrm{mM}$ and stored at $4^{\circ} \mathrm{C}$. A 1:10 dilution of this stock in DMSO was made prior to its addition to $\mathrm{KSOM}$. Concentrations of PPP ranging from 0.05 to $0.5 \mu \mathrm{M}$ were prepared by serial dilution in KSOM.

\section{Zygote collection}

All procedures involving the use of animals were conducted in accordance with the Australian Code of Practice for Use of Animals in Research and approved by the University of Sydney Animal Care and Ethics Committee. Quackenbush Swiss (QS) mice (Animal Resource Centre, Perth, Australia) were used in this study and housed under a 12-hour light: 12-hour dark cycle. Female mice (4-10 weeks old) were superovulated by intraperitoneal injection of 10 International Units (I.U.) of pregnant mare serum gonadotrophin (PMSG; Intervet, Sydney, Australia) followed by intraperitoneal injection of 10 I.U. of human chorionic gonadotrophin (hCG; Intervet) 48 hours later. They were then paired with a stud male QS mouse (10-30 weeks old) overnight. The presence of a vaginal plug the following day indicated successful mating.

Female mice were euthanized by cervical dislocation 20-22 hours after hCG administration in order to recover zygote stage embryos. Zygotes were isolated from the oviducts into Hepes mod-HTF $\left(37^{\circ} \mathrm{C}\right)$ and then isolated from their surrounding cumulus cells by addition of approximately $100 \mu \mathrm{l}$ of $1 \mathrm{mg} / \mathrm{ml}$ hyaluronidase (Sigma-Aldrich) to the Hepes mod-HTF $(3 \mathrm{ml})$. Zygotes were then collected and washed in at least three changes of KSOM. In some instances embryos were cultured in the absence of their zonae pellucidae. In these cases zygotes, were treated for approximately 3 minutes with pronase $(10 \mathrm{mg} / \mathrm{ml})$ in Hepes mod-HTF until their zonae thinned, upon which they were washed in KSOM to completely dissociate the zygote from its zona.

\section{Embryo culture}

Embryos were cultured from the zygote stage in round bottom 96-well plates (Corning, NY, USA) containing $\mathrm{KSOM}$, pre-warmed to $37^{\circ} \mathrm{C}$ and equilibrated at $5 \% \mathrm{CO}_{2}$ for a minimum of two hours. High density culture conditions were achieved by culturing embryos in groups of 10-15 at a concentration of 1 embryo per $1 \mu \mathrm{l}$ medium. Embryos cultured at high density were in contact with one another. Low density culture conditions were achieved by culturing embryos individually in $100 \mu \mathrm{l}$ or $300 \mu \mathrm{l}$ medium. Embryos were incubated at $37^{\circ} \mathrm{C}$ and $5 \% \mathrm{CO}_{2}$. Embryos were scored according to their developmental stage (2-cell, 4-cell, 8-cell, compacted, morula, cavitated and blastocyst) each day at 24 hour intervals. Blastocyst development and hatching from the zona pellucida was noted on day six of development (144 hours post-hCG). The medium was not changed over the six day period.

\section{Cell number and apoptotic cell determination}

In order to differentially stain inner cell mass and trophectoderm cells, cultured blastocysts were transferred to a 1:10 dilution of anti-mouse lymphocyte antibody (Sigma-Aldrich) in Hepes mod-HTF for 8 minutes at $37^{\circ} \mathrm{C}$ allowing the antibody to bind to the trophectoderm cells. Blastocysts were then washed five times in Hepes mod-HTF before being transferred to a 1:10 dilution of guinea pig complement containing propidium iodide (25 $\mu \mathrm{g} / \mathrm{ml}$; Sigma-Aldrich) in Hepes mod-HTF for 4 minutes. Blastocysts were then washed 5 times in Hepes mod-HTF before being fixed in $4 \%$ PFA for 15 minutes. Blastocysts were then quickly rinsed in PBS $+1 \mathrm{mg} / \mathrm{ml}$ PVA and mounted in $5 \mu \mathrm{l}$ Vectashield containing $1.5 \mu \mathrm{g} / \mathrm{ml}$ 4',6'-diamidino2-phenylindole (DAPI; Vector Laboratories, Burlingame, CA, USA).

Nuclei were visualised using confocal microscopy (LSM 510 Meta, Carl Zeiss, Germany) using a $405 \mathrm{~nm}$ laser and $561 \mathrm{~nm}$ laser at $40 \mathrm{X}$ objective. A Z-stack was taken through the embryo, at $2.5 \mu \mathrm{m}$ intervals, enabling nuclei at all depths to be visualised. Cells were counted manually using LSM Image Browser software (Carl Zeiss). Nuclei stained only with DAPI and not propidium iodide were counted as Inner cell mass (ICM) cells (Fig. S2). For total cell counts and counts of apoptotic nuclei blastocysts were fixed in 4\% PFA for 15 minutes, rinsed in PBS + PVA and mounted in $5 \mu \mathrm{l}$
Vectashield + DAPI. Nuclei were visualised using confocal microscopy (for total cell counts) or epifluorescence (for apoptotic nuclei, Olympus IX51).

\section{Western blotting}

Blastocysts and morula were isolated from the reproductive tract 94 hours after hCG administration and cultured in KSOM for 24 hours until fully expanded. Blastocysts were then treated with $100 \mathrm{ng} / \mathrm{ml}$ IGF1 for 10 minutes, washed in cold PBS $+1 \mathrm{mg} / \mathrm{ml}$ PVA and transferred to lysis buffer (Cell Signaling, Beverly, MA, USA) + 1 mM PMSF (Sigma) before being snap frozen in liquid nitrogen. Protein samples were diluted with $6 x$ Laemmli buffer (35 mM Tris- $\mathrm{HCl}, \mathrm{pH} 6.8,10.28 \%$ (w/v) SDS, 36\% (v/v) glycerol, $0.05 \%$ $(\mathrm{w} / \mathrm{v})$ bromophenol blue; Sigma) and heated for 5 minutes at $100^{\circ} \mathrm{C}$. Proteins were electrophoresed on an $8 \%$ SDS-polyacrylamide gel, transferred to a nitrocellulose transfer membrane (Hy-BLOT, Australia) and then incubated in blocking buffer (Odyssey; Li-cor Biosciences, Lincoln, NE, USA) overnight at $4^{\circ} \mathrm{C}$ with gentle shaking. Membranes were probed overnight at $4^{\circ} \mathrm{C}$ in blocking buffer $+0.1 \%$ Tween-20 with 1:500 rabbit anti-phospho-Akt (Ser473) (Cell signaling, \#4058) or 1:1000 mouse anti- $\alpha$-tubulin (Sigma, \#T9026). Membranes were washed in Tris-buffered saline + Tween 20 (TBST; $10 \mathrm{mM}$ Tris- $\mathrm{HCl}, \mathrm{pH} 7.6,150 \mathrm{mM} \mathrm{NaCl}$ and $0.1 \%$ Tween 20) and subsequently incubated for 2 hours with either 1:4000 Donkey anti-Rabbit IRDye 800CW (Odyssey) or 1:4000 Donkey anti-Mouse IRDye 680LT (Odyssey). Proteins were visualised using the Odyssey infrared imager.

\section{Time-lapse microscopy to determine effects of PPPon spindle structure}

Oocytes were isolated from the oviducts of unmated female mice 14-17 hours after $\mathrm{hCG}$ administration into Hepes mod-HTF $\left(37^{\circ} \mathrm{C}\right)$. Oocytes were treated with $0.4 \mathrm{nM} \alpha$-Chymotrypsin (Sigma-Aldrich) in Hepes mod-HTF at $37^{\circ} \mathrm{C}$ until their surrounding cumulus cells and zonae pellucidae were removed. Oocytes were incubated with $10 \mu \mathrm{g} / \mathrm{ml}$ Hoechst 33258 (Roche, Indianapolis, IN, USA) in Hepes mod-HTF at $37^{\circ} \mathrm{C}$ for 15 minutes. Oocytes were then treated with or without $0.5 \mu \mathrm{M}$ PPP for 6 hours. Oocytes were imaged over the 6 hour period using the CellVoyager ${ }^{\mathrm{TM}}$ CV1000 Confocal Scanner System (Yokogawa, Tokyo, Japan) using $405 \mathrm{~nm}$ laser at $5 \%$ laser power. A Z-stack was taken through the embryo, at $1.7 \mu \mathrm{m}$ intervals, every 5 minutes. Oocytes were processed for immunofluorescence at the 6 hour end-point. Additionally a set of control and treatment oocytes were kept in the incubator at $37^{\circ} \mathrm{C}$ where they were processed for immunofluorescence at the 6 hour end-point.

\section{Immunofluorescence of oocytes and embryos}

Oocytes and embryos were fixed with $4 \%$ paraformaldehyde for 15 minutes at room temperature and then washed in PBS + poly-vinyl alcohol (PVA). They were then permeabilised with PBS + PVA + 0.3\% Triton-X-100 for 15-30 minutes and then washed three times, with the final wash lasting 10 minutes. They were then blocked in PBS + PVA + 0.1\% Tween-20 $+0.7 \%$ BSA for $15-30$ minutes. Primary antibodies were diluted in PBS + PVA $+0.1 \%$ Tween-20 + 0.7\% BSA. Oocytes were incubated $1: 200$ mouse anti- $\beta$-tubulin (MAB3408; Millipore; Billerica, MA, USA) overnight at $4^{\circ} \mathrm{C}$. Embryos were incubated in 1:100 mouse anti- $\beta$-tubulin and 1:100 mouse anti- $\alpha$-tubulin (T9026; Sigma-Aldrich) overnight at $4^{\circ} \mathrm{C}$. Following primary antibody incubation oocytes/embryos were washed in PBS + PVA+Tween-20 + BSA three times, with the final wash lasting 10 minutes. They were then incubated in secondary antibody (1:200 Alexa Fluor 594 goat anti-mouse IgG; A-11005; Molecular Probes ${ }^{\circledR}$ Invitrogen, Carlsbad, CA, USA) for two hours at room temperature. They were then washed in PBS + PVA + Tween-20 + BSA three times, with the final wash lasting 10 minutes. Then they were transferred to $5 \mu \mathrm{l}$ Vectashield + DAPI. Nuclei and secondary antibody fluorescence were visualised using confocal microscopy using a $405 \mathrm{~nm}$ and Argon laser (458, 477, 488 and $514 \mathrm{~nm}$ lines) at $40 \mathrm{X}$ objective.

\section{Statistical analysis}

Embryos/oocytes were randomly allocated into control and treatment groups. Chi-squared analysis of the effects of culture conditions on embryo development were performed on at least three pooled experiments unless 
stated otherwise and compared the control group to the treatment group. Cell counts from at least three pooled experiments were compared using unpaired t-tests for single comparisons or One-way ANOVA followed by Dunnett's multiple comparisons test (performed using GraphPad Prism version 6.00 for Windows, GraphPad Software, La Jolla California USA www.graphpad.com), for multiple comparisons to the control. Cell counts were expressed as the mean \pm standard error of the mean (SEM). Western blot analysis was performed on groups of 150 embryos $(n=3)$. Densitometry was performed using Image-J software. The optical density of pAkt bands was normalised to $\alpha$-tubulin and expressed relative to the control, followed by statistical analysis using unpaired t-tests.

\section{Declaration of interest}

The authors declare that there is no conflict of interest that could be perceived as prejudicing the impartiality of the research reported.

\section{Funding}

This work was supported by funds from the University of Sydney and the Bosch Institute.

\section{Acknowledgements}

We would like to thank Dr. Louise Cole (Advanced Microscopy Facility, Bosch Institute, University of Sydney) for help with confocal microscopy. We would also like to thank Olympus, Australia for their loan of the CellVoyager $^{T M}$ CV1000 Confocal Scanner System.

\section{References}

BEDZHOV, I., LISZEWSKA, E., KANZLER, B. and STEMMLER, M.P. (2012). Igf1r signaling is indispensable for preimplantation development and is activated via a novel function of E-cadherin. PLoS Genet 8: e1002609.

BRISON, D.R. (2000). Apoptosis in mammalian preimplantation embryos: Regulation by survival factors. Human Fertility 3: 36-47.

CHI, M.M.Y., SCHLEIN, A.L. and MOLEY, K.H. (2000). High Insulin-Like Growth Factor 1 (IGF-1) and Insulin Concentrations Trigger Apoptosis in the Mouse Blastocyst via Down-Regulation of the IGF-1 Receptor. Endocrinology 141: 4784-4792.

ERBACH, G.T., LAWITTS, J.A., PAPAIOANNOU, V.E. and BIGGERS, J.D. (1994). Differential growth of the mouse preimplantation embryo in chemically defined media. Biol Reprod 50: 1027-1033.

GIRNITA, A., GIRNITA, L., DEL PRETE, F., BARTOLAZZI, A., LARSSON, O. and AXELSON, M. (2004). Cyclolignans as inhibitors of the insulin-like growth factor-1 receptor and malignant cell growth. Cancer Res 64: 236-242.

HALET, G., VIARD, P. and CARROLL, J. (2008). Constitutive Ptdlns(3,4,5)P3 synthesis promotes the development and survival of early mammalian embryos. Development 135: 425-429.

HARDY, K. and SPANOS, S. (2002). Growth factor expression and function in the human and mouse preimplantation embryo. J Endocrinol 172: 221-236.

HARVEY, M.B. and KAYE, P.L. (1992). Insulin-like growth factor-1 stimulates growth of mouse preimplantation embryos in vitro. Mol Reprod Dev 31: 195-199.

HERRLER, A., EINSPANIER, R. and BEIER, H.M. (1997). Binding of IGF-I to preimplantation rabbit embryos and their coats. Theriogenology 47: 1595-1607.

HERRLER, A., VON WOLFF, M. and BEIER, H.M. (2002). Proteins in the extraembryonic matrix of preimplantation rabbit embryos. Anat Embryol (Berl) 206: 49-55.

HEYNER, S., SHI, C.Z., GARSIDE, W.T. and SMITH, R.M. (1993). Functions of the IGFs in early mammalian development. Mol Reprod Dev 35: 421-426.

HO, Y., WIGGLESWORTH, K., EPPIG, J.J. and SCHULTZ, R.M. (1995). Preimplantation development of mouse embryos in KSOM: Augmentation by amino acids and analysis of gene expression. Mol Reprod Dev 41: 232-238.
INZUNZA, J., DANIELSSON, O., LALITKUMAR, P.G., LARSSON, O., AXELSON, M., TOHONEN, V., DANIELSSON, K.G. and STAVREUS-EVERS, A. (2010). Selective insulin-like growth factor-I antagonist inhibits mouse embryo development in a dose-dependent manner. Fertil Steril 93: 2621-2626.

KELLEHER, J.K. (1977). Tubulin binding affinities of podophyllotoxin and colchicine analogues. Mol Pharmacol 13: 232-241.

LANE, M. and GARDNER, D.K. (1992). Effect of incubation volume and embryo density on the development and viability of mouse embryos in vitro. Hum Reprod 7: 558-562.

LIU, J.-P., BAKER, J., PERKINS, A.S., ROBERTSON, E.J. and EFSTRATIADIS, A. (1993). Mice carrying null mutations of the genes encoding insulin-like growth factor I (Igf-1) and type 1 IGF receptor (Igf1r). Cell 75: 59-72.

LOIKE, J.D., BREWER, C.F., STERNLICHT, H., GENSLER, W.J. and HORWITZ, S.B. (1978). Structure-activity study of the inhibition of microtubule assembly in vitro by podophyllotoxin and its congeners. Cancer Res 38: 2688-2693.

MARKHAM, K.E. and KAYE, P.L. (2003). Growth hormone, insulin-like growth factor I and cell proliferation in the mouse blastocyst. Reproduction 125: 327-336.

MELIN, J., LEE, A., FOYGEL, K., LEONG, D.E., QUAKE, S.R. and YAO, M.W. (2009). In vitro embryo culture in defined, sub-microliter volumes. Dev Dyn 238: 950-955.

MONTAG, M., KOLL, B., HOLMES, P. and VAN DER, V. (2000). Significance of the number of embryonic cells and the state of the zona pellucida for hatching of mouse blastocysts in vitro versus in vivo. Biol Reprod 62: 1738-1744.

O'NEILL, C. (1997). Evidence for the requirement of autocrine growth factors for development of mouse preimplantation embryos in vitro. Biol Reprod 56:229-237.

O'NEILL, C. (1998). Autocrine mediators are required to act on the embryo by the 2-cell stage to promote normal development and survival of mouse preimplantation embryos in vitro. Biol Reprod 58: 1303-1309.

O'NEILL, C. (2008). The potential roles for embryotrophic ligands in preimplantation embryo development. Hum Reprod Update 14: 275-288.

PARIA, B.C. and DEY, S.K. (1990). Preimplantation embryo development in vitro: cooperative interactions among embryos and role of growth factors. Proc Natl Acad Sci USA 87: 4756-4760.

RIBAS, R.C., TAYLOR, J.E., MCCORQUODALE, C., MAURICIO, A.C., SOUSA, M.R and WILMUT, I. (2006). Effect of Zona Pellucida Removal on DNA Methylation in Early Mouse Embryos. Biol Reprod 74: 307-313.

RILEY, J.K., CARAYANNOPOULOS, M.O., H. WYMAN, A., CHI, M., RATAJCZAK, C.K. and MOLEY, K.H. (2005). The PI3K/Akt pathway is present and functional in the preimplantation mouse embryo. Dev Biol 284: 377-386.

SMITH, R.M., GARSIDE, W.T., AGHAYAN, M., SHI, C.Z., SHAH, N., JARETT, L. and HEYNER, S. (1993). Mouse preimplantation embryos exhibit receptor-mediated binding and transcytosis of maternal insulin-like growth factor I. Biol Reprod 49:1-12.

SPANOS, S., BECKER, D.L., WINSTON, R.M. and HARDY, K. (2000). Anti-apoptotic action of insulin-like growth factor-I during human preimplantation embryo development. Biol Reprod 63: 1413-1420.

STOJANOV, T., ALECHNA, S. and O'NEILL, C. (1999). In-vitro fertilization and culture of mouse embryos in vitro significantly retards the onset of insulin-like growth factor-II expression from the zygotic genome. Mol Hum Reprod5: 116-124.

STOJANOV, T. and O'NEILL, C. (2001). In vitro Fertilization Causes Epigenetic Modifications to the Onset of Gene Expression from the Zygotic Genome in Mice. Biol Reprod 64: 696-705

SUMMERS, M.C. and BIGGERS, J.D. (2003). Chemically defined media and the culture of mammalian preimplantation embryos: historical perspective and current issues. Hum Reprod Update 9: 557-582.

SUZUKI, H., TOGASHI, M., ADACHI, J. and TOYODA, Y. (1995). Developmental ability of zona-free mouse embryos is influenced by cell association at the 4-cell stage. Biol Reprod 53: 78-83.

VELAZQUEZ, M.A., ZARAZA, J., OROPEZA, A., WEBB, R. and NIEMANN, H. (2009). The role of IGF1 in the in vivo production of bovine embryos from superovulated donors. Reproduction 137: 161-180.

WHITTEN, W.K. (1956). Culture of tubal mouse ova. Nature 177: 96. 


\section{Further Related Reading, published previously in the Int. J. Dev. Biol.}

The role of Akt signalling in the mammalian ovary

Sandra Cecconi, Annunziata Mauro, Valerio Cellini and Felice Patacchiola

Int. J. Dev. Biol. (2012) 56: 809-817

http://dx.doi.org/10.1387/ijdb.120146sc

c-Jun N-terminal kinase activity supports multiple phases of 3D-mammary epithelial acinus formation

Sara McNally, Emmett McArdle, Emer Gilligan, Silvia Napoletano, Malgorzata Gajewska, Orla Bergin, Sarah McCarthy, Jacqueline Whyte, Alessandro Bianchi, Julianne Stack and Finian Martin

Int. J. Dev. Biol. (2011) 55: 731-744

http://dx.doi.org/10.1387/ijdb.113374sm

Developmental cell biology of human villous trophoblast: current research problems John D. Aplin

Int. J. Dev. Biol. (2010) 54: 323-329

http://dx.doi.org/10.1387/ijdb.082759ja

Comparative analysis of TGF beta s, BMPs, IGF1, msxs, fibronectin, osteonectin and bone sialoprotein gene expression during normal and in vitro-induced odontoblast differentiation.

C Bègue-Kirn, A J Smith, M Loriot, C Kupferle, J V Ruch and H Lesot

Int. J. Dev. Biol. (1994) 38: 405-420

http://www.intjdevbiol.com/web/paper/7848824

Growth factors and proto-oncogenes in early mouse embryogenesis and tumorigenesis. K Pavelic, N P Slaus and R Spaventi

Int. J. Dev. Biol. (1991) 35: 209-214

http://www.intjdevbiol.com/web/paper/1814404

5 yr ISI Impact Factor $(2011)=2.959$
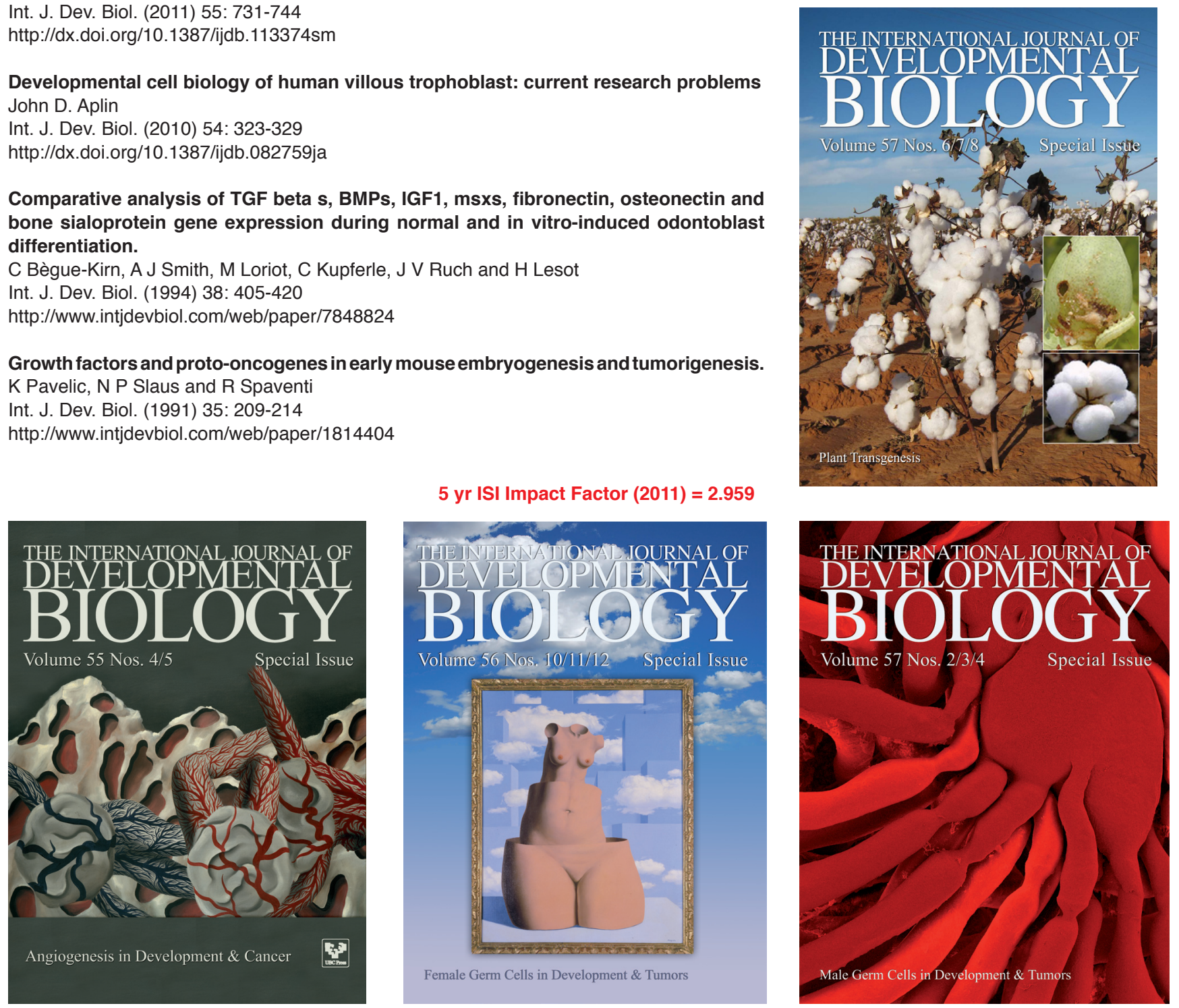